\title{
Meyer's surgical procedure for the treatment of lip carcinoma
}

Received: 22 July 2003 / Accepted: 2 January 2004 / Published online: 5 March 2004

(C) Springer-Verlag 2004

\begin{abstract}
Lip carcinomas are generally treated by surgery. A reconstruction is often required if the resected segment exceeds one-third of the lip. Meyer's plasty is an alternate way of reconstructing the lower or upper lip. The aim of this study is to describe the technique, its indications and results. A retrospective review of all patients who underwent a Meyer's plasty in our institution is presented. Twenty-four consecutive patients were treated in Lausanne for T1 and T2 lip carcinomas between 1983 and 2001. Primary surgery associated with Meyer's plasty was performed in all cases. Data were collected from the medical records, and eight patients were recalled for clinical evaluation. The oncological, functional and aesthetic results were analyzed. The 5-year local control was $100 \%$. Three patients developed metachronous lymph node metastasis. No patient died from the disease. A hindering microstomy was found in three cases, and two patients suffered from temporary oral leakage. No speech difficulty was encountered. The aesthetics was described as satisfying or good in $87 \%$ of the patients. Meyer's plasty following lip surgery of the upper or lower lip allows an aesthetic and functional one-stage reconstruction without compromising the oncological outcome.
\end{abstract}

Keywords Lip $\cdot$ Carcinoma $\cdot$ Reconstruction $\cdot$ Meyer's plasty $\cdot$ Aesthetics

This study was presented at the annual meeting of the Swiss Association for Otolaryngology and Head and Neck Surgery in Pontresina, Switzerland, 20-22 June 2002.

Y. Jaquet (四) P. Pasche $\cdot$ E. Brossard $\cdot$ P. Monnier

F. J. W. Lang

Department of Otolaryngology and Head and Neck Surgery,

Centre Hospitalier Universitaire Vaudois,

1011 Lausanne, Switzerland

Tel.: +41-213-142700, Fax: +41-213-142706,

e-mail: yves.jaquet@hospvd.ch

\section{Introduction}

Up to $25 \%$ of cancers of the oral cavity are lip carcinomas $[21,25]$. They mainly occur in male patients who are middle aged or older. Most of the malignancies are squamous cell carcinomas and $95 \%$ of them develop on the lower lip. The major risk factor involved is cumulative sun light exposure [21]. Other factors may include social status or smoking habits [21]. Lip carcinomas are often diagnosed at an early stage. Although surgery and radiation therapy can both be considered as acceptable treatment options, surgery remains the treatment of choice. Radiation therapy may play a role for those patients who will not accept surgery or who carry a high operative risk. Either choice offers equal local control $(>90 \%)$ and specific survival $(>80 \%)[20,22]$. Cervical lymph node metastasis (synchronous or metachronous) is found in $10-20 \%$ of patients. A prophylactic selective neck dissection of level I, II and III groups of lymph nodes (supraomohyoid neck dissection) is usually planned for large tumors $(>3 \mathrm{~cm})$, for tumors with little differentiation or for local recurrences [25].

Traditionally, full-thickness excisions are performed, although for superficial lesions, Moh's micrographic surgery is the most widespread alternative. A plain wedgeshaped resection is recommended for lesions leaving a defect up to one-third of the lip length [8, 24]. Larger excisions require a proper reconstruction procedure, according to the location of the tumor. Recognition of the functional and aesthetic consequences is of the utmost importance when discussing treatment options, particularly for the upper lip $[4,8]$.

An original reconstruction technique was described in 1964 by Rodolphe Meyer, a surgeon from Lausanne, Switzerland. It was first published under the name of his assistant, P. Hertig [10]. The technique was adapted from the procedures of Bernard [2] and Ginestet [9] and is contemporary with Fries' procedure [7]. Meyer's plasty allows the resection of medial or lateral tumors of both the upper and lower lip with a transversal extension of the lesion involving up to $80 \%$ of the total lip length $[5,14,15,16,17,18]$. 

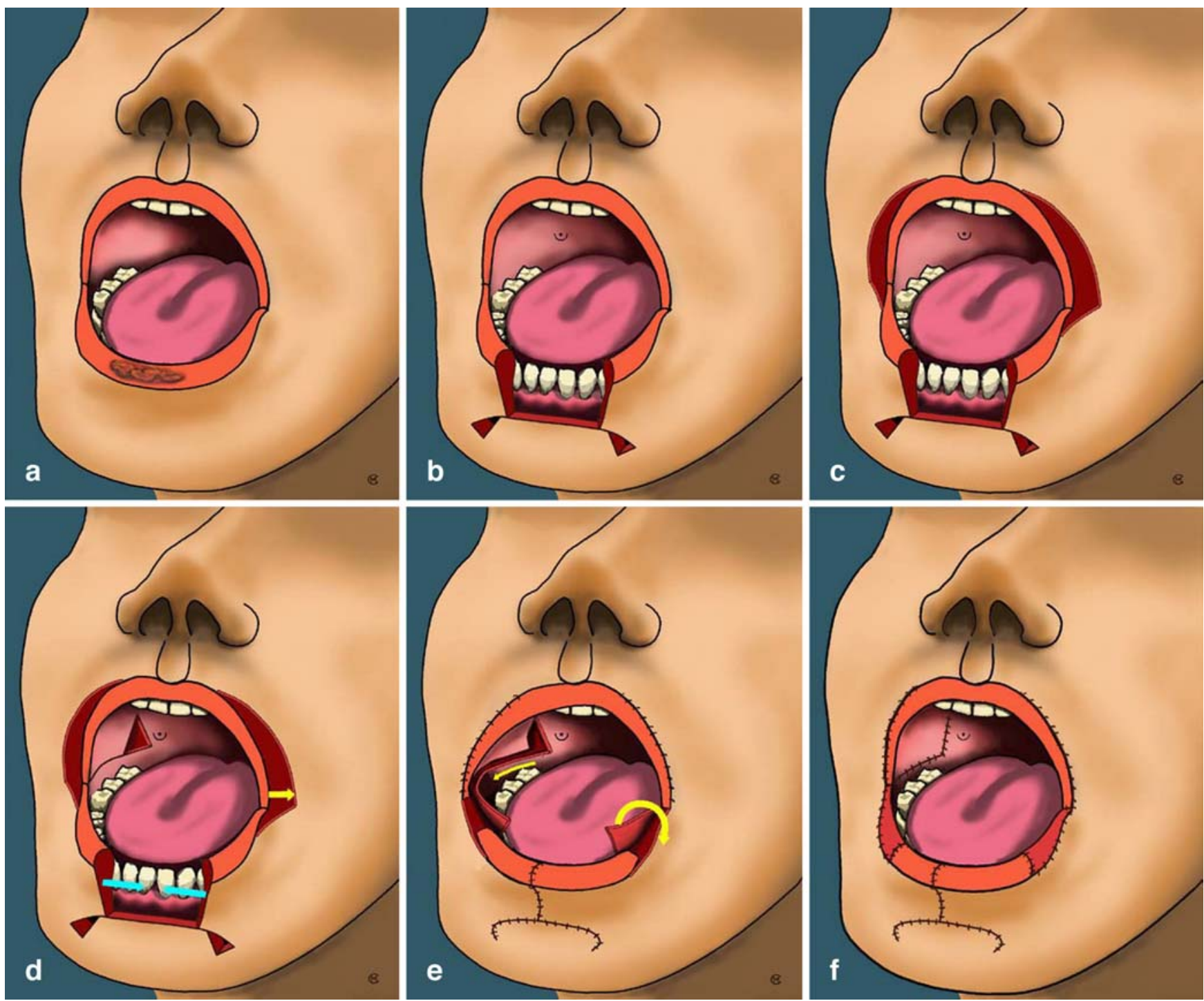

Fig. 1 Meyer's plasty: course of the procedure for a lower lip tumor. a Tumor. b Tumor excision. c Commissuroplasty: triangular cutaneous excision. d Mucosal flap incision and lower lip closure, blue arrows. e Eversed mucosal flap, yellow arrows. f End result with scars along the white line and labiomental crease

For near total excision of the lower lip, a Meyer plasty can also be associated with a flap of Abbe [16, 17].

\section{Materials and methods}

Surgical technique

\section{Lower lip $[5,10,14,15,16,17,18,24]$}

The tumor (Fig. 1a) resection is trapezium-shaped, through the whole thickness of the lower lip reaching the gingivo-jugal sulcus. The cutaneous incision line at its base is prolonged to either side of the chin, ending at both ends in Burow's triangles (Fig. 1b).

Commissuroplasty is usually performed on both sides (Fig. 1c). However, if the defect is lateral and of small dimension, then this step may be undertaken unilaterally. When done bilaterally, commissuroplaty allows easier tissue movement and hence scar tension release. A skin triangle is excised above the vermillion of the upper lip with its base located next to the commissure, the medial edge following precisely the white line of the lip. The lateral wedge will correspond to the new oral commissure. When performed unilaterally, the triangle base should match the length of the defect. For bilateral commissuroplasty, each triangle's base will measure half of the length of the defect.

For reconstruction of the mucosal lining, buccal mucosa is released by creating two lateral advancement flaps (Fig. 1d). The oral incision line goes from the commissure to the orifice of the Stenon's duct. At both ends, Burow's triangles are excised in order to prepare the advancement flaps. Enough mucosa is obtained to cover the lateral part of the new vermilion.

The remaining lateral segments of the lower lip are brought together, thus achieving median closure of the lip with a minimum of tension (Fig. 1d, blue arrow). A nude area on both sides of the lower lip remains, corresponding to the former triangle base (Fig. 1e).

A critical step of the procedure is the dissection of the orbicularis muscle. During the prior triangular skin resection and the mucosal incision, the muscle is carefully prepared. Its fibers are stretched and laterally displaced to the new oral commissure. Damaging the orbicularis oris muscle must be avoided to preserve its function.

The intraoral mucosal flap is then medially advanced. Its superior border must be everted and pulled forward over the upper edge of the lip in order to cover the nude area and form the new lateral vermilion (Fig. 1e, yellow arrows). The end result is shown in Figs. 1f and 2. 
Fig. 2 Inverted Meyer's plasty: medial upper lip tumor. a Tumor resection with sickleshaped skin excisions along the alar bases. Inverted commissuroplasty (compare to Fig. 1c and d). b End result with scars hidden along the alar base and the white line
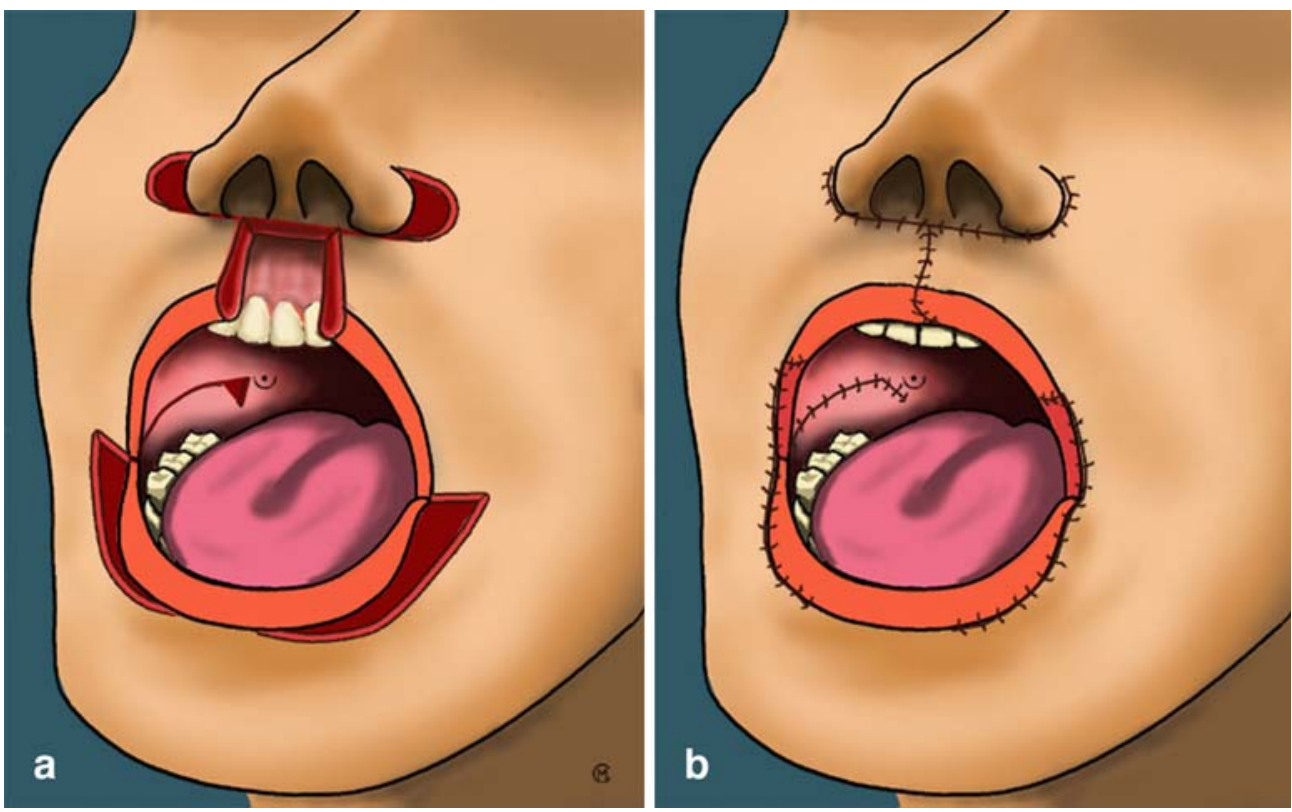
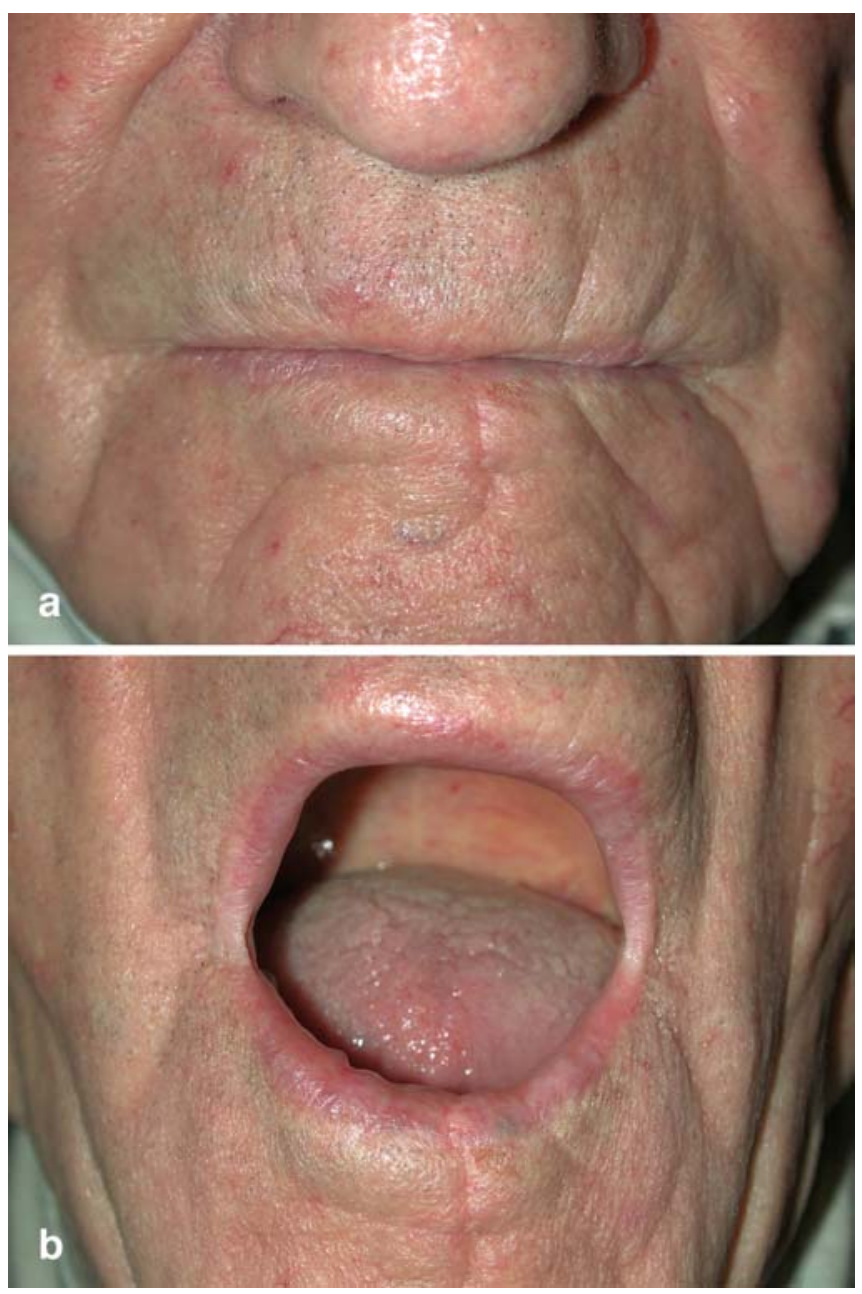

Fig. 3 Lower lip reconstruction in a 62-year-old male patient. Good aesthetic and functional result. a Only the median vertical scar of the lower lip is still visible, but merges with the surrounding wrinkles. b Symmetrical and sufficient mouth opening

\section{Upper lip}

The corresponding technique adapted for the upper lip (inverted Meyer's plasty) follows the same principles as previously described $[5,14,17]$ (Figs. 3 and 4). Tumor resection is trapeziumshaped, through the whole thickness of the upper lip, reaching the gingivolabial sulcus and the nasolabial fold upward. Sickle-shaped skin excisions along the alar base are performed on both sides. Their width should measure half of the surgical defect length in order to release sufficient tissue to close the upper lip. Commissuroplasty is carrried out on one or both sides, depending on the size and location of the tumor. The skin excision is done along the white line of the lower lip, and its base should correspond to half of the length (bilateral commissuroplasty) or the total length (unilateral commissuroplasty) of the surgical defect. Mucosal flap is constituted to cover the new lateral upper lip. The oral incision ends in an inverted Burow's triangle located anterior to the Stenon's duct orifice. With advancement and eversion of the mucosal flap, enough mucosa is obtained to line the nude area of the lateral upper lip.

\section{Patients}

This retrospective study included 24 consecutive patients who underwent the excision of a lip carcinoma with reconstruction by a Meyer's plasty at the Lausanne University Hospital between January 1983 and December 2001. The clinical and pathological data are shown in Table 1 . The only inclusion criterion was the surgical reconstruction technique. No patient treated by a Meyer's plasty was excluded from the study. The treatment options (surgery or radiotherapy) were decided during an oncologic multidisciplinary meeting. All patients were then included in the same standard oncologic follow-up. The data were collected from the medical records.

Eight out of the 24 patients made themselves available for a follow-up clinical examination, which included functional evaluation, aesthetic evaluation and photographic documentation. Among the remaining 16 patients, 5 could not be contacted, 3 patients declined the recall, and 8 patients were deceased (many years after surgery). Those later deaths consequently did not influence the 5-year survival rates. The mean follow-up time was 5.1 years (range: 2 months to 16 years). The systematic follow-up allowed oncologic analysis of the whole series of patients. The TNM classification was determined according to the 1997 UICC criteria [26]. Specific survival, local and loco-regional control and median follow-up 

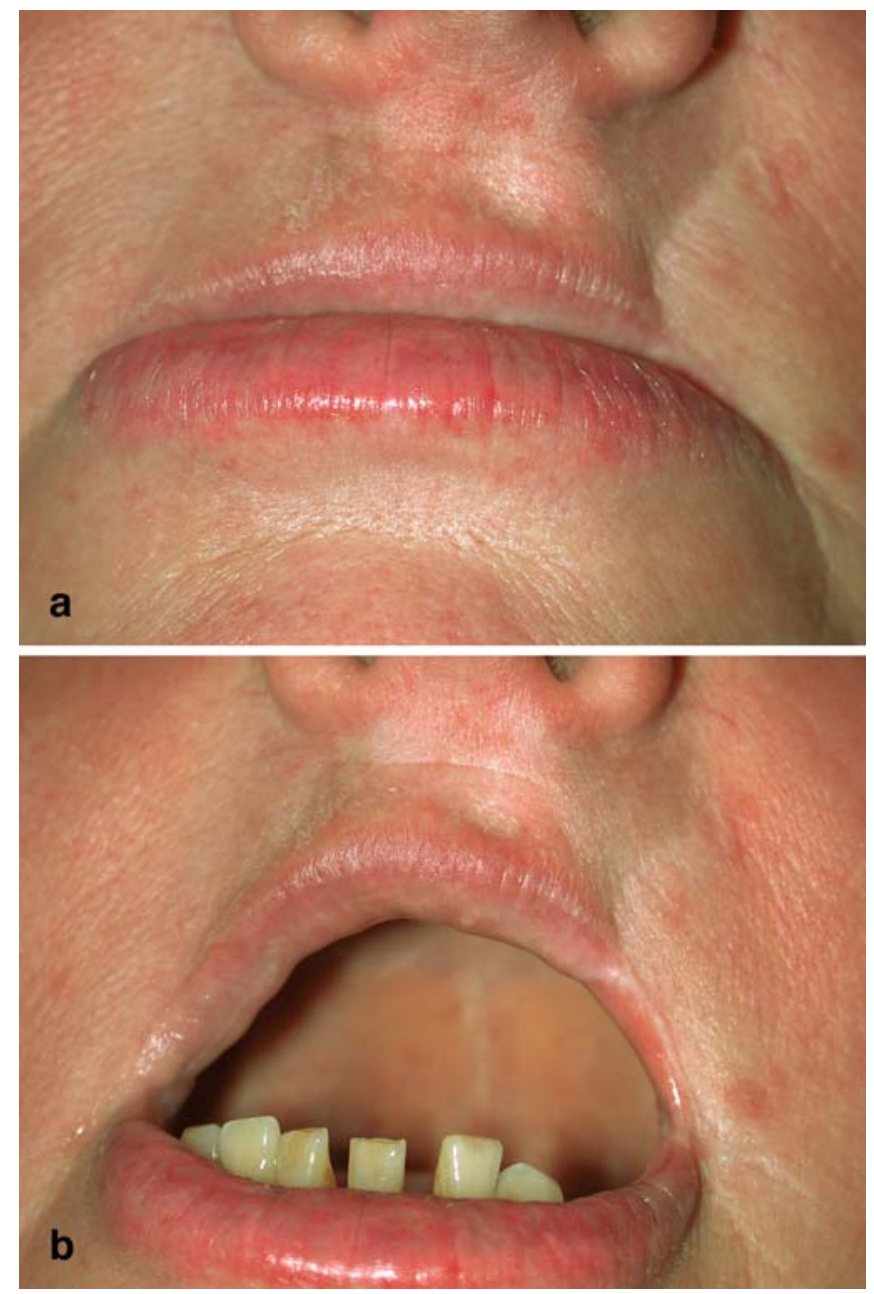

Fig. 4 Left upper lip reconstruction in a 57-year-old female patient. Satisfying aesthetic and functional result. a The left vertical scar of the upper lip is still visible and the new lateral upper lip is slightly thinner than on the opposite side. b Sufficient but asymmetrical mouth opening

were calculated with the Kaplan-Meier actuarial method. No further statistical analysis was performed because of the small number of patients.

Functional results were evaluated for the global pool of patients. The detailed medical records allowed the detection of a hindering microstomy (the patient complained that the mouth opening was too small), oral leakage or speech difficulties.

The aesthetic result was only evaluated for the eight patients who could be clinically examined because the medical records did not offer consistent and complete information concerning this point. Only one out of eight patients had an upper lip reconstruction. The patient's and the investigator's opinions were taken into account separately.

\section{Results}

Three patients presented with an early partial dehiscence of the commissuroplasty between the 1st and 5th postoperative day. One of them had to be revised. No deaths or other serious complications were reported.
Table 1 Clinico-pathological data

\begin{tabular}{ll}
\hline Variable & $n$ or $\%$ \\
\hline Analysis & \\
$\quad$ Medical record & $24 / 24$ \\
Clinical examination & $8 / 24$ \\
Mean age (min - max) & $66(33-89)$ \\
Gender & \\
Male & $21(87 \%)$ \\
Female & $3(13 \%)$ \\
Tumor stage & \\
T1 & $21(87 \%)$ \\
T2 & $3(13 \%)$ \\
Nodal stage & \\
N0 & $21(87 \%)$ \\
N1 & $1(\mathrm{~T} 1)$ \\
N2a & $1(\mathrm{~T} 1)$ \\
N2c & $1(\mathrm{~T} 2)$ \\
Histology & \\
Squamous cell carcinoma & $20(83 \%)$ \\
Verrucous carcinoma & 2 \\
Basal cell carcinoma & 1 \\
Undifferentiated carcinoma & 1 \\
Site & \\
Upper lip (right-midline-left) & $4(1-0-3)(17 \%)$ \\
Lower lip (right-midline-left) & $20(12-5-3)(83 \%)$ \\
\hline
\end{tabular}

All tumors were of stage T1 or T2. The transversal diameter of the lesions was between 1 and $4 \mathrm{~cm}$. Tumor excisions could all be performed with secure, tumor-free surgical margins. No prophylactic neck dissection was undertaken in the N0 patients. Synchronous lymph node metastases were found in three patients $(13 \%)$. A functional neck dissection was then performed simultaneously, and for two of them, cervical radiotherapy was added because of capsular rupture. Those three patients did not show any loco-regional recurrence. Metachronous nodal metastasis was diagnosed in three other patients (13\%), 3 months, 4 months and 3 years after the initial treatment, respectively. They were treated by functional neck dissection, and for two cases, also with postoperative cervical radiotherapy. These patients did not develop any loco-regional recurrence. The 5-year local control was $100 \%$, and the locoregional control was $84 \%$, with three nodal recurrences. No distant metastases were noted. No patient died because of the tumor (specific survival: 100\%), and the 5-year overall survival was $94 \%$ at median follow-up (Table 2).

Four patients showed functional disabilities in the early postoperative period (Table 2). Three of them suffered from a reduced mouth opening resulting in difficulty inserting and removing their dentures. In two cases, the microstomy was a consequence of the postoperative wound dehiscence. One of them could be solved by a surgical correction. The third patient with hindering microstomy showed an initial tumor of $4 \mathrm{~cm}$ of lateral extension (not including surgical margins), and Meyer's plasty might not have been the best treatment option. 
Table 2 Results

\begin{tabular}{ll}
\hline Variable & $n$ or $\%$ \\
\hline Oncological outcome & At median follow-up \\
Local control & $100 \%$ \\
Loco-regional control & $84 \%$ \\
Specific survival & $100 \%$ \\
Overall survival & $94 \%$ \\
Functional disabilities & $4 / 24$ \\
Hindering microstomy & $3 / 24$ \\
Oral incompetence & $2 / 24$ \\
Speech difficulty & $0 / 24$ \\
Aesthetic evaluation & Good or satisfactory \\
Patient & $100 \%$ \\
Examiner & $87.5 \%$ \\
\hline
\end{tabular}

Two patients presented with postoperative oral leakage. One of these two patients also had a microstomy: the microstomy as well as the oral leakage were secondary to the postoperative wound dehiscence. In the other patient, the etiology of the leakage remains unclear, but could be corrected with a single collagen injection into the commissure. Speech difficulties were not noted in any patient, neither in the short nor the long term.

At long-term follow-up, out of 24 patients, only 2 suffered a definitive disability, consisting of both microstomy and oral leakage in one case and microstomy alone in the other. Aesthetic results (Table 2) were evaluated as being either good or satisfactory in all cases according to the patient, while only in seven out of eight cases according to the examiner (Figs. 2 and 4). The patient with an unsatisfactory aesthetic result showed a vermillon fading on the side opposite to the tumor. This patient was one of the three patients suffering from postoperative wound dehiscence.

\section{Discussion}

The oncological outcome of this method is very satisfactory, but the small number of patients reviewed in this study has to be taken into account. Nevertheless, our control and survival rates are within the range of the published data $[20,21,22,25]$. At a median follow-up of 5 years, the actuarial local control rate was $100 \%$. Meyer's plasty therefore seems appropriate for the treatment of lip cancer. The only three recurrences were regional neck lymph nodes. These metachronous nodal metastases occurred initially in N0 patients. None of the NO patients in this series was treated with prophylactic neck dissection. The salvage procedure was efficient in all cases.

Only few functional evaluations with objective parameters have been found in the literature on reconstructive surgery of the lip [13, 19], thus allowing no valuable comparison of our results with those of other authors. Three out of 24 patients presented a postoperative hindering microstomy secondary to a postoperative dehiscence in two cases and to an improper use of the technique in one case (tumor size too large to perform a Meyer's plasty). Oral leakage was found in two patients and was also associated with a postoperative suture dehiscence in one case. The technique itself is therefore not responsible for these complications.

The aesthetic result was judged to be very satisfactory by the patients as well as by the examiners. Even so, all evaluation scales were subjective. The only patient with a poor aesthetic result suffered a postoperative wound dehiscence, and the method was not the cause of this outcome. Postoperative dehiscence is obviously the main feature influencing the final functional and aesthetic outcome. This can be prevented by releasing scar tensions by using a precise design of Burow's triangles, extensive mucosal undermining before commissuroplasty and adapted postoperative care. It is also of major importance to use the procedure only when properly indicated.

Many methods of lip reconstruction after large excisions have been reported, but few of them meet the actual requirements for a satisfactory functional and aesthetic outcome. Among the classical techniques available for lip repair, Bernard's procedure is well known for its good cosmetic outcome $[2,8]$. However, sectioning the orbicularis oris muscle leads to a variable loss of the sphincter function and consecutive oral incompetence. Webster's modification of Bernard's technique has the same drawbacks [8, 21]. The Step Technique described by Johanson has also gained wide acceptance for large resections of up to two-thirds of the lower lip [3,11]. Preservation of sensory and motor function is well achieved with a Karapandzic flap, which is indicated for medial lesions of both the lower and upper lip [12]. Large lateral defects up to one half of the lip length may also be repaired by an Abbe flap [1]. If the commissure is involved, then an Estlander flap may be used [6]. However, the flap's designs do not prevent an interruption of the orbicularis muscle, and the scars are not hidden along anatomic lines.

The Meyer's procedure should be considered because of its multiple advantages: the orbicularis oris muscle is preserved, thus allowing an optimal functional result; the scars are well hidden along the white line and labiomental crease, explaining the good aesthetics with natural looking oral commissures; because of an optimal mucosal lining, primary healing is achieved and deforming or retractile scars can be avoided; finally, Meyer's plasty is a onestage and time-saving procedure, which is particularly important for the elderly.

\section{Conclusion}

Reconstruction of the lip following tumor excision remains a challenge. The surgeon has to meet the goal of an acceptable aesthetic and functional result while having to observe safe surgical margins. Meyer's plasty fulfills these requirements by preserving the continuity of the orbicularis muscle and hiding scars along anatomic lines. The oncological outcome of this method is satisfactory. Meyer's plasty can be recommended as a safe and efficient technique to resolve $\mathrm{T} 1$ and $\mathrm{T} 2$ upper and lower lip 
tumors of a maximal lateral extension of $4 \mathrm{~cm}$, including resection margins.

Acknowledgements The authors thank Mr. Claude Marechaux for his contribution to the figures.

\section{References}

1. Abbe R (1898) A new plastic operation for the relief of deformity due to double harelip. Med Record 53:477-478

2. Bernard C (1853) Cancer de la lèvre inférieure opéré par un procédé nouveau. Bull Mem Soc Chir Paris 3:357-363

3. Blomgren I, Blomqvist G, Lauritzen C, Lilja J, Peterson L-E, Holmström $H$ (1988) The step technique for the reconstruction of lower lip defects after cancer resection: A follow-up study of 165 cases. Scand J Plast Reconstr Surg 22:103-111

4. Burget G, Menick F (1986) Aesthetic reconstruction of one half of the upper lip. Plast Reconst Surg 78:5

5. Draf W, Schauss F (1990) Lip reconstruction with Meyer plasty: a universal procedure? Facial Plast Surg 7:132-136

6. Estlander JA (1872) Eine Methode aus der einen Lippe Substanzverlust. Arch Klin Chir 14:622

7. Fries R (1971) The merits of Bernard's operation as an universal procedure for lower lip reconstruction after resection of carcinoma. Chir Plast 1:45-52

8. Gaylon SW, Frodel JL (2001) Lip and perioral defects. Otolaryngol Clin North Am 34:647-666

9. Ginestet G (1946) Reconstruction de toute la lèvre inférieure par des lambeaux naso-géniens totaux. Rev Odontol Stomatol $8: 28$

10. Hertig P (1965) Une nouvelle technique de reconstruction plastique de la lèvre inférieure. Pract Oto-Rhino-Laryngol 2:157166

11. Isaksson I, Johanson B (1967) Partial reconstruction of the lower lip. Panminerva Med 9:420

12. Karapandzic M (1974) Reconstruction of lip defects by local arterial flaps. Brit J Plast Surg 27:1
13. Lopez AC, Ruiz PC, Campo FJR, Gonzalez FD (2000) Reconstruction of lower lip defects after tumor excision: An aesthetic and functional evaluation. Otolaryngol Head Neck Surg 123: 317-323

14. Meyer R (1990) Lip surgery. Facial Plast Surg 7:126-131

15. Meyer R, Shapiro MA (1973) A technique for immediate reconstruction of the lower lip after ablation of tumour. Chir Plastica 2:1-16

16. Meyer R, Failat ASA (1982) New concept in lower lip reconstruction. Head Neck Surg 4:240-245

17. Meyer R, Mayer B, Perko D (1989) Rekonstruktion der Oberlippe mit der Mukosa-Eversions-Technik. Laryngo-Rhino-Otol 68:591-594

18. Poget M, Meyer R (1976) Modification d'une technique de reconstruction après résection totale ou subtotale de la lèvre inférieure. ORL 38:127-132

19. Stranc MF, Fogel M, Dische S (1987) Comparison of lip function: surgery vs radiotherapy. Br J Plast Surg 40:598-604

20. Veness M (2001) Lip cancer: important managment issue. Australas J Dermatol 42:30-32

21. De Visscher JGAM, Schaapveld MM, Otter R, Visser O, van der Waal I (1998) Epidemiology of cancer of the lip in the Netherlands. Oral Oncol 34:421-426

22. De Visscher JGAM, Botke G, Schakenraad JACM, van der Waal I (1999) A comparison of results after radiotherapy and surgery for stage I squamous cell carcinoma of the lower lip. Head Neck 21:526-530

23. Webster RC, Coffey RJ, Kelleher RE (1960) Total and partial reconstruction of the lower lip with innervated muscle-bearing flaps. Plast Reconstr Surg 25:360-371

24. Weerda H (1990) Surgery of lower lip defects. Facial Plast Surg 7:84-96

25.Zitsch RP, Lee BW, Smith RB (1999) Cervical lymph node metastases and squamous cell carcinoma of the lip. Head Neck 21:447-453

26. TNM Classification of Malignant Tumors (1997) Sobin LH, Wittekind C (eds) International union against cancer (UICC), 5th edn. Wiley-Liss Publishers, New York, pp 20-24 